\title{
Genome replication engineering assisted continuous evolution (GREACE) to improve microbial tolerance for biofuels production
}

Guodong Luan ${ }^{1,2}$, Zhen Cai ${ }^{*}$, Yin Li $i^{*}$ and Yanhe $\mathrm{Ma}^{3}$

\begin{abstract}
Background: Microbial production of biofuels requires robust cell growth and metabolism under tough conditions. Conventionally, such tolerance phenotypes were engineered through evolutionary engineering using the principle of "Mutagenesis followed-by Selection". The iterative rounds of mutagenesis-selection and frequent manual interventions resulted in discontinuous and inefficient strain improvement processes. This work aimed to develop a more continuous and efficient evolutionary engineering method termed as "Genome Replication Engineering Assisted Continuous Evolution" (GREACE) using "Mutagenesis coupled-with Selection" as its core principle.

Results: The core design of GREACE is to introduce an in vivo continuous mutagenesis mechanism into microbial cells by introducing a group of genetically modified proofreading elements of the DNA polymerase complex to accelerate the evolution process under stressful conditions. The genotype stability and phenotype heritability can be stably maintained once the genetically modified proofreading element is removed, thus scarless mutants with desired phenotypes can be obtained.

Kanamycin resistance of $E$. coli was rapidly improved to confirm the concept and feasibility of GREACE. Intrinsic mechanism analysis revealed that during the continuous evolution process, the accumulation of genetically modified proofreading elements with mutator activities endowed the host cells with enhanced adaptation advantages. We further showed that GREACE can also be applied to engineer n-butanol and acetate tolerances. In less than a month, an E. coli strain capable of growing under an n-butanol concentration of $1.25 \%$ was isolated. As for acetate tolerance, cell growth of the evolved E. coli strain increased by 8 -fold under $0.1 \%$ of acetate. In addition, we discovered that adaptation to specific stresses prefers accumulation of genetically modified elements with specific mutator strengths.
\end{abstract}

Conclusions: We developed a novel GREACE method using "Mutagenesis coupled-with Selection" as core principle. Successful isolation of E. coli strains with improved n-butanol and acetate tolerances demonstrated the potential of GREACE as a promising method for strain improvement in biofuels production.

Keywords: Mutagenesis coupled-with Selection, GREACE, Genome replication engineering, Continuous evolution, Strain improvement

\footnotetext{
* Correspondence: caiz@im.ac.cn; yli@im.ac.cn

${ }^{1}$ CAS Key Laboratory of Microbial Physiological and Metabolic Engineering, Institute of Microbiology, Chinese Academy of Sciences, No. 1 West Beichen Road, Chaoyang District, Beijing 100101, China

Full list of author information is available at the end of the article
} 


\section{Background}

Efficient microbial production of biofuels from renewable resources requires robust cell growth and stable metabolism under tough industrial conditions, represented by inhibitory components in substrates and toxic products [1,2]. Microbial tolerance to these inhibitory environmental factors is a complex phenotype usually controlled by multiple genes [3,4], and thus is difficult to be engineered by targeted metabolic engineering approaches [5]. Instead, such complex phenotypes can be more effectively improved by evolutionary engineering approaches [6]. Examples of evolutionary engineering include successive passage for metabolic evolution [7-9], physical and chemical mutagenesis [10], global transcription machinery engineering $[2,11]$, artificial transcription factors engineering [12,13], and ribosome engineering [14]. All these methods use "Mutagenesis followed-by Selection" as core principle, meaning that firstly introducing genetic diversity by spontaneous mutations, exogenous mutagens, or genetic perturbations, followed by selection of desired phenotypes [6]. Using such methods, iterative rounds of mutagenesis-selection and frequent manual interventions are often required, resulting in discontinuous and inefficient strain improvements, as shown in Figure 1A.

To address the discontinuity of the existing evolutionary engineering approaches and improve the engineering efficiency, we devised a novel method termed as "Genome Replication Engineering Assisted Continuous Evolution, GREACE”, which uses "Mutagenesis coupledwith Selection" (Figure 1B) as its core principle. The key element of this novel method is to introduce in vivo continuous mutagenesis mechanisms into microbial cells that are subsequently subjected to continuous selective conditions. Mutagenesis and selection can therefore be coupled to minimize manual interventions, thus providing possibilities to develop a continuous and efficient phenotypes-improving process (Figure 1B). Practically, in vivo continuous mutagenesis can be achieved by introducing genetic perturbations into genome replication machinery so as to trigger inaccurate genome replications. Hypermutable cells with significant genome diversities can therefore be obtained. Offspring cells with mutated genomes that survived the increased selective pressures will be selected during the continuous enrichment processes, and the genomic feature of evolved cells with improved phenotypes can be stably maintained once the elements triggering the inaccurate genome replication are removed from the individually isolated cells.

In this work, we proved the concept of GREACE and tested the efficiency of GREACE using Escherichia coli as a model. Genetically modified proofreading elements of the DNA polymerase complex ( $\varepsilon$ subunit encoded by $d n a Q$ gene) were used to trigger perturbations on genome replication for in vivo continuous mutagenesis. We firstly proved the feasibility and intrinsic mechanisms of GREACE by improving kanamycin resistance of $E$. coli. Secondly we show that n-butanol and acetate tolerances of $E$. coli, two important microbial tolerance characteristics, can also be efficiently improved using this method. E. coli mutants obtained through the GREACE process showed significantly improved tolerances to n-butanol and acetate, demonstrating the potential of GREACE as an effective and universal approach to improve tough physiological traits required by biofuels production. Furthermore, we discovered a phenomenon that adapting to

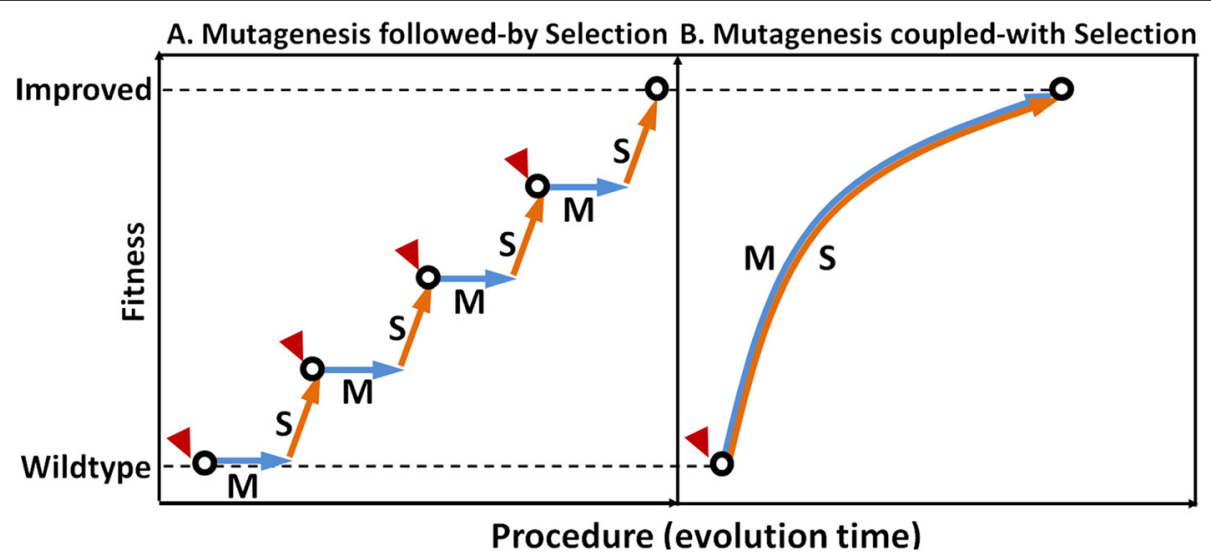

Figure 1 Comparisons between the principle of "Mutagenesis followed-by Selection" and the principle of "Mutagenesis coupled-with Selection". (A) Traditional "Mutagenesis followed-by Selection" principle was usually performed by iterative rounds of mutagenesis and selection. Exogenous mutagens or genetic manipulations were required for mutagenesis and the following selection manipulations isolated cells with improved phenotypes, which could be used in next rounds of "mutagenesis-selection". (B) As for "Mutagenesis coupled-with Selection" principle, the two steps are synchronized, so that iterative and lengthy manual interventions are greatly simplified, leading to a continuous and efficient strain improvement process. "S" represents for "selection", " $M$ " represents for "mutagenesis", and red triangles represent for manual interventions such as mutagen treatments or genetic manipulations or selections for improvement phenotypes. 
specific environments calls for specific genetically modified proofreading elements, which may provide insights into understanding and application of evolutionary engineering for strain improvement.

\section{Results}

Procedure of the "Genome Replication Engineering Assisted Continuous Evolution"

The core idea of GREACE is to introduce genetic perturbations into genome replication machinery, so that in vivo continuous mutagenesis can be coupled with simultaneous phenotypes selection. A typical flowchart of GREACE is described in Figure 2. To achieve an inaccurate genome replication, genetic perturbations on genome replication machinery are generated by constructing a mutant library of a proofreading element (PE) gene, designated as PEM-lib. Subsequently, the PEM-lib is transformed into the wildtype strain. Inaccurate genome replication will be triggered in cells containing PEs with decreased proofreading activities, thus continuously generating offspring cells containing different genomic mutations. Notably, the diversity of the PEM-lib will help to generate cells with various genome replication mutation rates and mutation type preferences, ensuring the highest genomic diversity in offspring cells.
When cells containing a genetically modified PEs are cultivated under gradually increased selective pressures, only the offsprings with accumulated adaptive mutations can survive and thus to be finally selected. Most importantly, the genome replication will return to regular state once the genetically modified PE is eliminated from the selected cells, so that the genomic features and the evolved phenotypes can be stably maintained.

\section{Proof of concept for GREACE by engineering kanamycin resistance of $E$. coli}

To prove the concept of GREACE, we took E. coli as a model strain and selected kanamycin resistance as a phenotype for testing. Among the various cellular proofreading elements including the genes responsible for base selection, exonucleolytic editing, MMR (Methyl-directed Mismatch Repair), and DNA repair [15-18], we chose the $d n a Q$ gene which encodes the $\varepsilon$ subunit of $E$. coli DNA polymerase III. This subunit is the only one with $3^{\prime}->5^{\prime}$ exonuclease activity in DNA polymerase III, the major DNA polymerase of $E$. coli genome replication, and it was reported to guarantee both the polymerization and errorediting process of DNA replication. Moreover, the strongest mutator phenotypes that have been known were resulted from deficiencies of the dnaQ gene $[17,19,20]$.

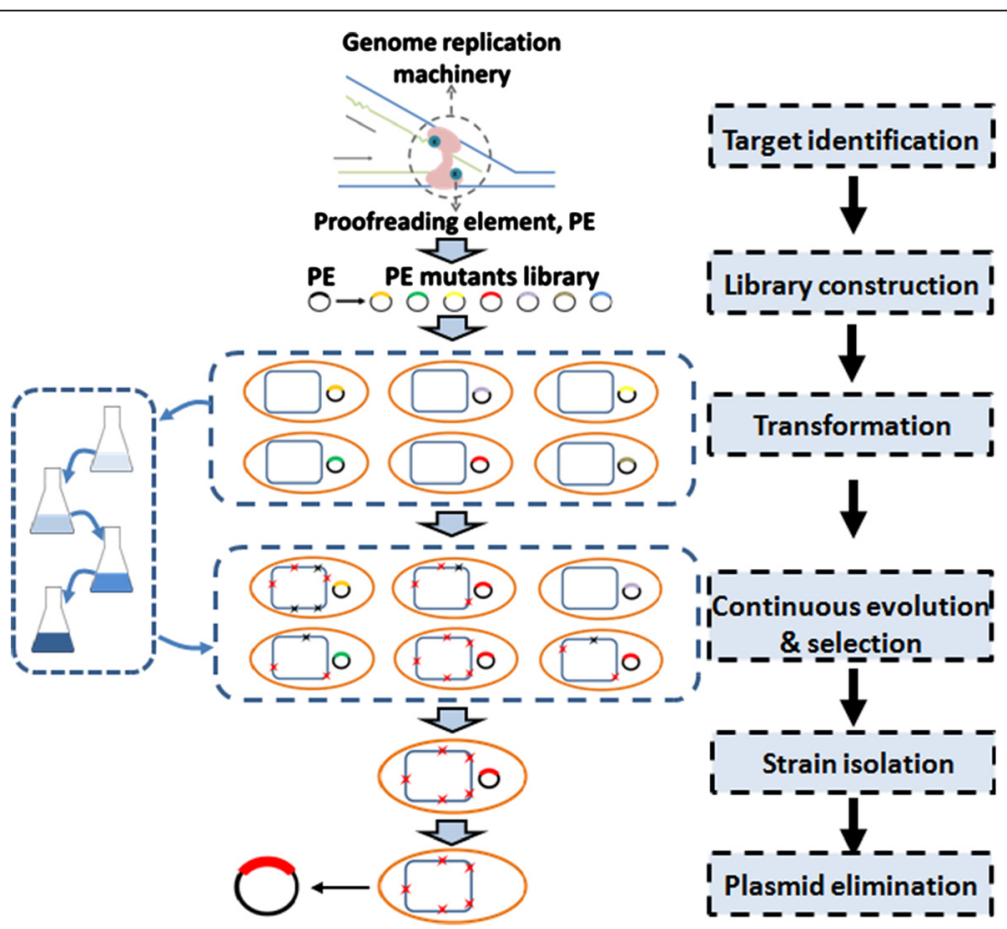

Figure 2 Flowchart of the Genome Replication Engineering Assisted Continuous Evolution (GREACE) method. Proofreading element (PE) of the genome replication machinery was selected to generate a mutant library (PEM-lib). For phenotype-improvement, PEM-lib was transformed into host cells, and cultivated in conditions with gradually increased selective strengths (media in flasks with colours from light blue to dark blue). Evolved strains with the most beneficial and adaptive mutation accumulations (red sparks represented for beneficial mutations and black sparks for detrimental mutations) would show the best adaption advantages and dominate the PEM-lib populations. Genetically modified PE mutant would be eliminated from the evolved strains to stabilize the obtained genotypes and phenotypes. 
Thus, $d n a Q$ was selected as the proofreading element to be engineered.

A mutant library of $d n a Q$ gene (pQ-lib) with the size of $10^{6}$ and average mutation rates of 2-3 amino acids per gene product, was constructed by error-prone PCR. $\mathrm{pQ}$-lib, together with the control vectors $\mathrm{pQ}$-dnaQ (carrying wildtype $d n a Q$ ), and the empty vector $\mathrm{pUC18}$, was transformed into $E$. coli cells, respectively. All cultures were serially transferred in media containing serial concentrations of kanamycin $(15 \mu \mathrm{g} / \mathrm{ml}, 100 \mu \mathrm{g} / \mathrm{ml}$, $200 \mu \mathrm{g} / \mathrm{ml}$, and $300 \mu \mathrm{g} / \mathrm{ml} ; \mathrm{X} \mu \mathrm{g} / \mathrm{ml}$ kanamycin will be designated as KanX). As shown in Figure 3A, no obvious growth differences were observed among three groups grown in Kan15. Upon increasing the kanamycin concentrations, growth and adaptation advantage of the pQ-lib group became obvious. Finally, the pQ-lib group was able to grow in Kan300, while the pUC18 and pQdnaQ groups failed to grow in Kan200 and Kan100, respectively, suggesting the pQ-lib group had a stronger adaptability.

To understand why pQ-lib group showed such adaptation advantages, cultures containing $10^{8}$ cells were collected from pQ-lib, pUC18, and pQ-dnaQ carrying strains grown at each kanamycin concentration and spread onto plates containing higher kanamycin concentrations (cells from Kan0, Kan15, Kan100, and Kan200 liquid culture were spread on plates containing Kan15,
Kan100, Kan200, and Kan300, respectively). As shown in Figure 3B, growths of the three groups in Kan0 did not make difference on generation of Kan15 resistant colonies. Growth of the pQ-lib group in Kan15 liquid culture generated more Kan100 resistant colonies than that of the controls, similar results were observed for pQ-lib group grown in Kan100 and Kan200. This suggested that pQ-lib group was able to generate kanamycin-resistant cells more rapidly.

To investigate the dynamic changes of the diversity of pQ-lib during the evolution process, 30 colonies of the pQ-lib group were randomly picked at the end of cultivations at each kanamycin concentration. Plasmids were extracted from these colonies and the dnaQ mutants therein were sequenced. As shown in the pie charts of Figure $3 \mathrm{~B}$, the diversity of $\mathrm{pQ}$-lib decreased dramatically, and one mutant termed as dnaQ KR5-2 was enriched, from undetectable level in Kan0 to 100\% in Kan200.

To verify whether the observed adaptation advantages were endowed by dnaQ KR5-2, plasmid pQ-dnaQ-KR52 was retransformed into fresh $E$. coli cells and cultivated under kanamycin stress. As expected, E. coli cells carrying dnaQ KR5-2 indeed exhibited increased evolution speed and adaptability. In Kan15 and Kan100, the dnaQ KR5-2 carrying strain grew to $\mathrm{OD}_{600}$ of $1.02( \pm 0.07)$ and $0.28( \pm 0.05)$ after cultivation for 48 hours, which were 3-4 fold higher than those of the

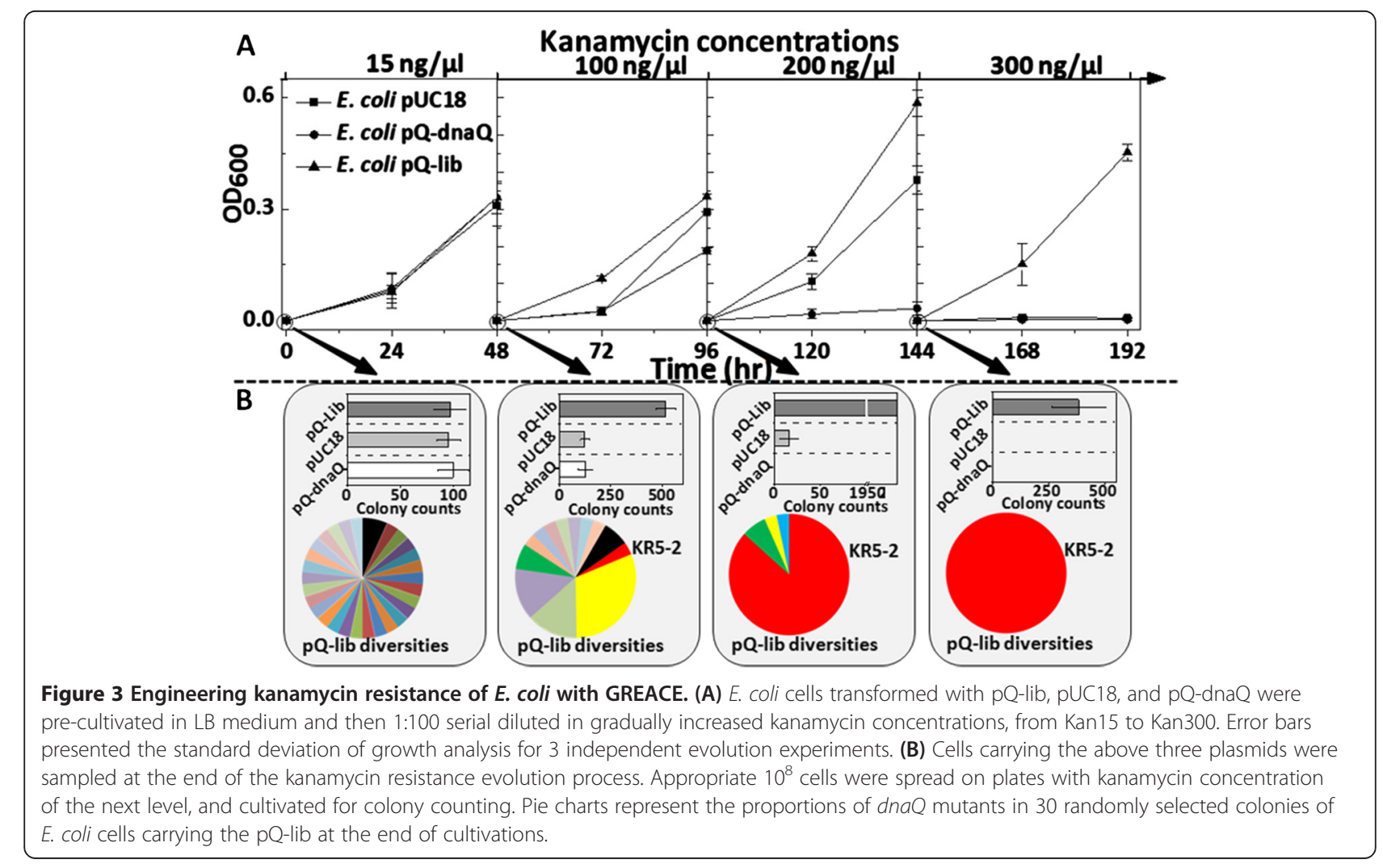


two control strains carrying pQ-dnaQ and pUC18 (Additional file 1: Figure S1). These results confirmed that adaptation advantages of $d n a Q$ KR5-2 under stressful conditions.

To quantitatively evaluate and compare the rates of generating genomic DNA mutations (termed as mutation rates for short) by different $d n a Q$ mutants, we chose the widely used rifamycin resistant colony generation frequency as an indirect indicator [21-23]. Mutation rates determination based on this indicator showed that $E$. coli cells carrying pQ-dnaQ-KR5-2 exhibited a 317-fold increased mutation rate than that of the wildtype control, confirming that introducing genetic perturbation into genome replication machinery indeed triggered inaccurate genome replication.

According to the design principle of GREACE, phenotypes obtained by GREACE should be stable and heritable. To confirm the stability of kanamycin resistance, plasmid pQ-dnaQ-KR5-2 was eliminated from the kanamycin resistant strain $E$. coli KR1. We found that the kanamycin resistance could be well maintained after plasmid elimination (Additional file 1: Figure S2).

\section{GREACE can be successfully applied to engineer n-butanol and acetate tolerances}

Recently, E. coli has been proved to be a promising host for biobutanol production [24-26]. However, n-butanol tolerance of $E$. coli is a bottleneck hampering further increase of n-butanol titer, as n-butanol is highly toxic to microbial cells. Many efforts have been made to improve and analyze butanol tolerance of $E$. coli [13,27-30]. We then wanted to test if GREACE can be used to improve n-butanol tolerance of $E$. coli, a tougher physiological trait comparing with kanamycin resistance. We applied a similar procedure to engineering n-butanol tolerance E. coli cells carrying pQ-lib were serially transferred in media containing gradually increased n-butanol concentrations. Considering that improvement of complex traits such as n-butanol tolerance might require accumulation of much more adaptive mutations [31,32], the pQ-lib carrying cells were transferred in each n-butanol concentrations for three times, upon full growth, before transferring to the next higher butanol concentration.

Figure 4A showed that $\mathrm{pQ}$-lib carrying cells exhibited a better adaptability to the increased n-butanol concentrations. After continuous cultivations and selections, pQ-lib group could grow in medium containing n-butanol concentration of $1.25 \%$ ( $\mathrm{vol} / \mathrm{vol}$, the same below), while n-butanol concentrations of $0.875 \%$ and $0.75 \%$ were lethal to the E. coli cells carrying pUC18 and pQ-dnaQ, respectively. The dnaQ mutant dominating the n-butanol tolerance evolution process was isolated and termed as dnaQ BR1. Further analysis revealed that $d n a Q$ BR1 endowed the host with a 2839-fold increased mutation rate, much higher than that of $d n a Q$ KR5-2 selected under kanamycin stress.

After plasmid elimination, an E. coli strain, designated as BT12, was obtained. Growth assay revealed that E. coli BT12 performed growth advantages upon n-butanol challenge (Figure 4B). At an n-butanol concentration of $0.75 \%$, growth of $E$. coli BT12 reached a doubled $\mathrm{OD}_{600}$ value after 48 hours cultivation compared with the wild type strain. When n-butanol concentration reached $1.25 \%$, no growth was detected for the wildtype, while E. coli BT12 was still able to grow (25\% $\mathrm{OD}_{600}$ value of that under no n-butanol stress). n-Butanol shock experiments revealed that E. coli BT12 showed a higher cellular stability and tolerance under an extreme lethal n-butanol concentration. After exposure to $2 \%$ n-butanol for 1 hour, the survival rate of E. coli BT12 was over 100-fold higher than that of the wildtype cells (Additional file 1: Figure S3). We further tested the stability of the n-butanol tolerance of $E$. coli BT12. After frozen at $-80^{\circ} \mathrm{C}$ for 2 weeks and serially transferred for 30 passages, the n-butanol tolerance of $E$. coli BT12 could be well maintained, indicating that good genetic stability and traits heritability can be retained upon GREACE (Additional file 1: Figure S4).

Acetate tolerance is another important trait for biofuels production. Concentrated acetate in hemicelluloses hydrolysates severely inhibited growth and metabolism of microbial cells, thus restricting conversion and production efficiency [33-35]. GREACE was also successfully applied to improve $E$. coli tolerance to acetate. As shown in Figure $4 \mathrm{C}$, the inhibitory effects of acetate on the evolved cells were sharply reduced after transferring $E$. coli cells carrying $\mathrm{pQ}$-lib in gradually increased acetate concentrations for 24 days. pQ-lib carrying cells successfully adapted to an acetate concentration of $0.105 \%$ ( $\mathrm{vol} / \mathrm{vol}$, the same below), while the control could hardly grow in a lower concentration of $0.09 \%$. After plasmid elimination, an E. coli mutant AR5 was obtained. AR5 cells showed an 8-fold increased $\mathrm{OD}_{600}$ as compared to that of the wildtype strain when grown in the presence of $0.1 \%$ acetate (Figure 4D). The dnaQ mutant dominating the acetate tolerance evolution process was isolated and termed as AR6-1, endowing host E. coli cells with an 87-fold increased mutation rate.

\section{Characterization of dnaQ mutants selected from different conditions}

Three different dnaQ mutants (KR5-2, BR1, and AR6-1), which endowed the host with different mutation rates, were enriched during the evolution process against increased concentrations of kanamycin, n-butanol, and acetate. To investigate whether these enriched $d n a Q$ mutants were the optimal that could always be enriched 


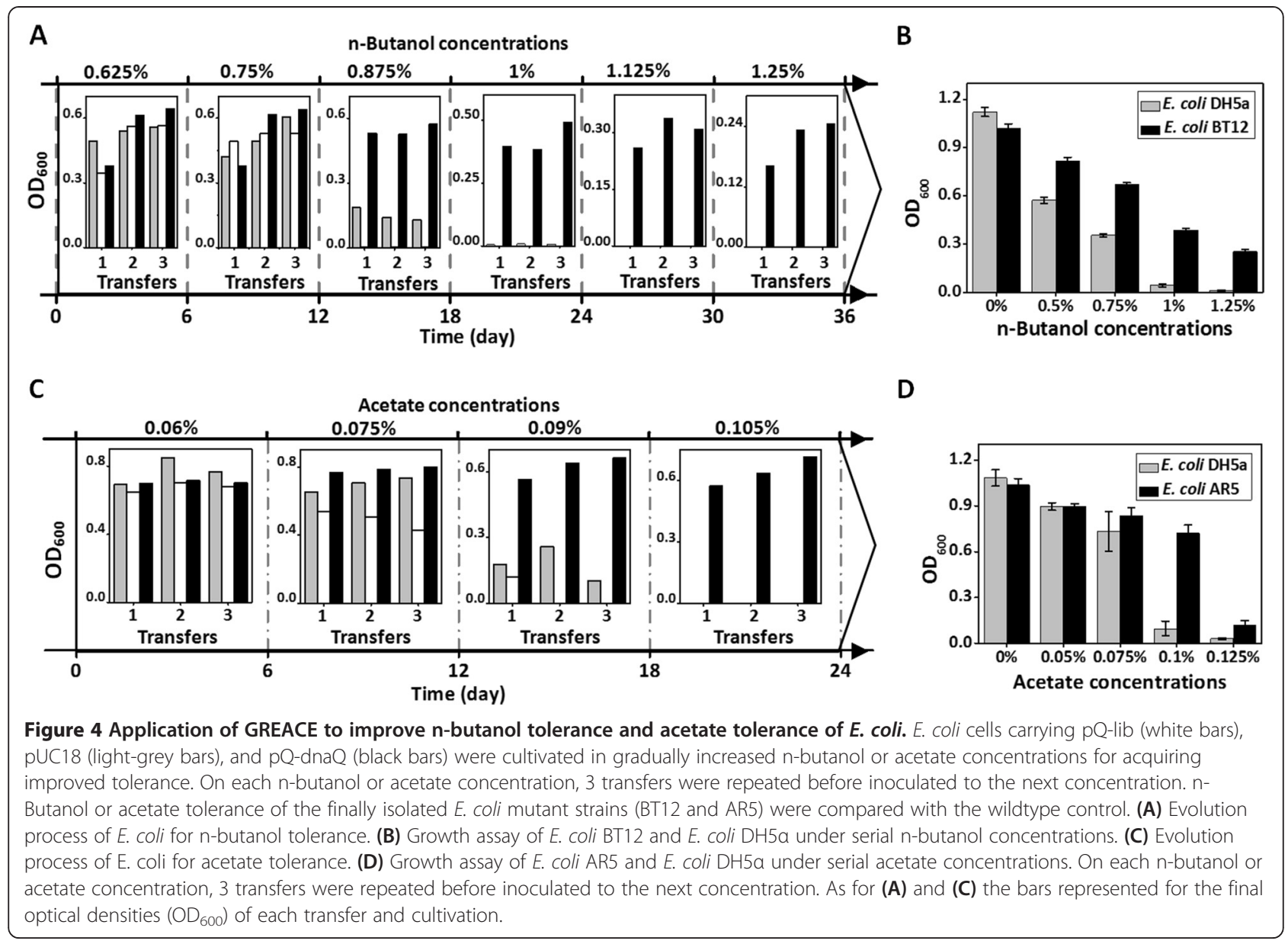

in independent evolution processes, we performed parallel experiments for acquiring kanamycin resistance, nbutanol tolerance, and acetate tolerance. The finally enriched $d n a Q$ mutants from each process were isolated, sequenced, and analyzed. In the parallel experiments for acquiring kanamycin resistance, nine different dnaQ mutants were isolated from 10 independent evolution processes. This suggests that the evolution process for acquiring kanamycin resistance is not dependent on one preferable dnaQ mutant. Similar results were also observed in parallel processes for acquiring n-butanol tolerance (12 parallel experiments) or acetate tolerance (10 parallel experiments). All dnaQ mutants isolated were different. Sequences of the $d n a Q$ mutants isolated were shown in Figure 5B. As expected, all of the selected mutants endowed the host cells with elevated mutation rates, ranging from 7.7 folds (dnaQ KR4-1) to 2839 folds (dnaQ BR1) (Figure 5A), which indicated the genetically modified $d n a Q$ mutants enriched in GREACE could serve as endogenous mutators to accelerate evolution.

We determined the mutation rates of all 31 dnaQ mutants isolated from parallel experiments, and grouped them into three types based on the increment of the mutation rates over that of the wildtype $d n a Q$ gene (termed as mutator strength for short): weak mutators (1-100 folds), medium mutators (100-1000 folds), and strong mutators ( $>1000$ folds). As shown in Figure $5 \mathrm{~A}$, nbutanol stress tended to select strong mutators. A large proportion (5/12) elevated mutation rates of host cells by over 1000 folds, while no weak mutators were found. Acetate stress tended to select weak mutators, as most $(6 / 10)$ of them falling into the weak group. Kanamycin stress tends to select dnaQ mutants with medium mutation rates, as the majority (7/9) showed medium strengths. This suggested that acquiring desired physiological traits required assistance of mutators with specific mutator strength. Calculation of average strengths of the mutators obtained in acquiring n-butanol tolerance (BtlR group, 959.8-fold increase), kanamycin resistance (KanR group, 375.3-fold increase), and acetate tolerance (AceR group, 249.4-fold increase) also supported this indication. Sequencing analysis revealed a positive relationship between the average mutator strengths and average amino acids substitution numbers in each $d n a Q$ mutant. Among the BtlR group, an average of 3.9 amino acids substitutions 


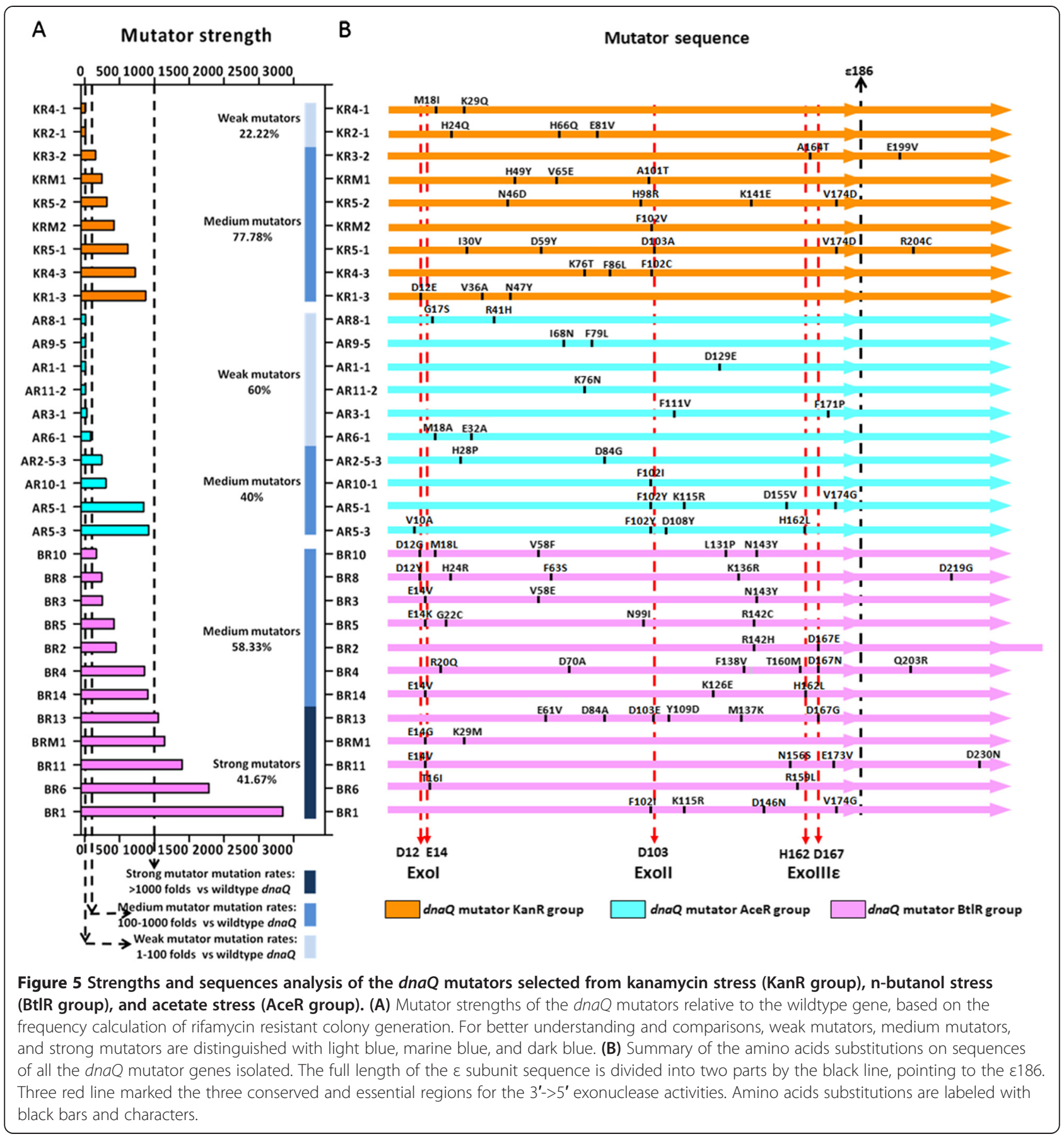

were found in each mutant, while the substitutions for KanR and AceR group were 2.9 and 2.1, respectively.

Analysis of the locations of the amino acids substitutions promoted understanding of the decreased proofreading activities of the selected $d n a Q$ mutants. Many efforts have been made to disclose the relationship between sequence and structure characteristics of the $\varepsilon$ subunits encoded by dnaQ gene [16,36]. The $3^{\prime} \rightarrow 5^{\prime}$ exonuclease activities of the $\varepsilon$ subunit was mainly determined by the N-terminal 186 amino acids (designated as
ع186) [37]. In this study, most of the mutation (88 out of 94) from all the 31 dnaQ mutants existed within the $\varepsilon 186$ region (Figure $5 \mathrm{~B}$ ), demonstrating that $\varepsilon 186$ played key roles in proofreading. Three conserved regions essential for proofreading, ExoI, ExoII, and ExoIII [38,39], have been recognized in $\varepsilon 186$. As expected, a large portion of amino acid substitutions of the selected dnaQ mutants were located in or quite close to these three regions, meaning high possibilities to disturb the natural proofreading function of the respective gene products. 


\section{Discussion}

The accurate genetic information transfer guarantees the genetic and phenotypic stability of organism, and it requires complex and precise mechanisms on multiple stages [18]. However, these hierarchal and precise mechanisms turn to be serious barriers for engineering complex phenotypes. To overcome such barriers, we developed GREACE, which implements a novel principle "Mutagenesis coupled-with Selection", and thus enables microbial evolution under stressful conditions in a continuous and efficient way.

As for microbes, expanding offspring genome diversities by elevating replication mutation rates to achieve rapid adaptation and competitive advantages in harsh environments has been discovered and verified both in vivo [40-42] and in silico [43,44]. Various natural mutator genes have been isolated and analyzed. However, some of them failed to work under some harsh conditions, e.g. high temperatures $[45,46]$. The GREACE method implants a pool of genetically modified proofreading elements into host cells to act as "evolution accelerator", thus provides multiple mutators with diverse characteristics which might guarantee accelerated evolution of host cells under diverse conditions.

Accelerating microbial evolution by mutators with elevated mutation rates during genome replication have been reported previously [47-49]. All these studies used a single specific mutator with a certain mutation rate. However, out work provided the first evidence that adaptation to specific conditions prefers accumulation of specific mutators, especially with specific mutator strengths. This finding was partially supported by Loh et.al [50], who found a narrow range of mutation rates (10-47 folds increase) dominated a designed laboratory survival competition. It has also been predicted by mathematical models that microbial survival and adaptation to different selective pressures might require different amounts and types of adaptive mutations, thus different mutagenesis strengths might be preferred [44]. These results benefit understanding and application of evolutionary engineering strategies for strain improvement, and further support the necessity for using GREACE method, which generated a library of mutators. Characterization of the $d n a Q$ mutants selected from GREACE under different conditions provided interesting clues to understand the intrinsic mechanisms of this novel approach. A universal $d n a Q$ mutant that can effectively improve all physiological traits might not exist, while a group of $d n a Q$ mutants may be helpful, as the most preferable $d n a Q$ mutants will be enriched during the evolution process.

In comparison with other phenotype-improving approaches generating global disturbances by introduction and maintaining of exogenous plasmids $[2,11,12]$, GREACE endows the microbes with stable and heritable phenotypes through scarless manipulations. Genotype stability and phenotype hereditability of the mutants can be maintained at any stage of evolution when the genetically modified proofreading elements are removed from the evolved mutants, and that provides great convenience for further genetic manipulation and application of mutants, e.g. introduction of the metabolic pathway for products synthesis.

Evolutionary engineering has been widely applied to optimize biofuels-producing related characteristics, especially for improvement of substrates utilization and inhibitors tolerant capacities [51-53]. GREACE provides a powerful new tool for evolutionary engineering, as the continuous and exhaustless genetic diversities generated by GREACE can provide nearly every possible solution while the synchronous selection will direct the most advantageous ones. Besides the cellular tolerance phenotypes engineered in this work, GREACE can also be expected to be applied for improving the metabolic capacities of biofuels in combination with the newly arising evolutionary metabolic engineering approach, which establishes the linkage between products synthesis and cell growth [54-56]. In addition, diverse improved phenotypes in mutants evolved by GREACE could be efficiently integrated in a single strain with multiple improved traits by methods like genome shuffling $[1,57]$. Hence, GREACE can be considered as a promising strain improving approach with wide application potentials.

\section{Conclusions}

A novel method termed as "Genome Replication Engineering Assisted Continuous Evolution" (GREACE), using "Mutagenesis coupled-with Selection" as core principle was developed to improve microbial tolerance for biofuels production. The GREACE method introduced an in vivo mutagenesis mechanism into microbial cells by introducing a group of genetically modified proofreading elements of the DNA polymerase $(\varepsilon$ subunit encoded by $d n a Q$ gene) to accelerate the evolution process under stressful conditions. The genotype stability and phenotype heritability can be stably maintained once the genetically modified proofreading element is removed, thus scarless mutants with desired phenotypes can be obtained.

GREACE was successfully applied to engineer $n$-butanol and acetate tolerances, two important physiological characteristics for biofuels production, demonstrating potentials of the GREACE method for strain improvement in this area. Furthermore, we discovered that adaptation of microbes to specific stresses prefers specific mutagenesis strengths, which may provide new insights on understanding and application of evolutionary engineering for strain improvement. 


\section{Methods}

\section{Strains and culture conditions}

E. coli DH5 $\alpha$ (TAKARA) was used for plasmids construction, phenotype evolution, and mutation rates evaluation. E. coli cells were grown aerobically in Luria-Bertani medium at $37^{\circ} \mathrm{C}$, unless there are special instructions. Antibiotics and kanamycin, n-butanol, and acetate were supplemented as required.

\section{Plasmid and library construction procedures}

The native $d n a Q$ gene and promoter fragment were both amplified from the E. coli DH5 $\alpha$ genomic DNA with Phusion DNA polymerase, using primer pairs dnaQ-F/ dnaQ-R, and dnaQProm-F/dnaQProm-R, respectively (Additional file 1: Table S1). The dnaQ fragment was cloned into the EcoRI and HindIII sites in pUC18 and the dnaQ promoter sequence was used to replace the lac promoter between the BspQI and EcoRI sites. The final plasmid was named as pQ-dnaQ.

Error-prone PCR was employed to construct a $d n a Q$ mutant library, with primer pairs of dnaQ-EP-F/dnaQEP-R (Additional file 1: Table S1). A standard error-prone PCR protocol was taken, and the mutation rate was controlled by adjusting concentrations of manganese and magnesium ions. The products was purified, digested and inserted into EcoRI and NdeI sites of the pQ-dnaQ plasmid to replace the wild type $d n a Q$ gene. The ligation system was transformed into $E$. coli DH5 $\alpha$ cells. After cultivation on agar plates, about $10^{6}$ transformants were obtained and scrapped off, and then the plasmids were extracted to generate a library named as pQ-lib. All enzymes used for DNA manipulation are from NEB.

\section{Evolution and phenotype selection}

E. coli $\mathrm{DH} 5 \alpha$ cells that were respectively transformed with pQ-lib, pQ-dnaQ, and pUC18 were transferred into fresh LB medium containing $100 \mu \mathrm{g} / \mathrm{ml}$ ampicillin (Amp100 for short) and grown overnight. Then the broth was serially transferred under gradually increased stress conditions. All of the evolution experiments in this part were performed with $10 \mathrm{ml}$ LB media supplemented with Amp100 and serial concentrations of kanamycin, nbutanol or acetate, and cultivated at $37^{\circ} \mathrm{C}$, unless there are special instructions. To evaluate cell densities, $\mathrm{OD}_{600}$ was monitored by microplate reader at $600 \mathrm{~nm}$ with a sample volume of $200 \mu \mathrm{l}$.

(i) Kanamycin resistance: $300 \mu \mathrm{l}$ of overnight culture broth of the transformants was inoculated into $10 \mathrm{ml} \mathrm{LB}$ medium containing Amp100 and Kan15, and cultivated for 2 days. Then $300 \mu$ of the culture broth (with an $\mathrm{OD}_{600}$ value adjusted to 0.2 by fresh LB medium) was transferred to LB medium containing Amp100 and Kan100, and cultivated for 2 days. The transfer and selection processes were repeated to LB media containing Kan200 and Kan300.

On each selection level, about $10^{8}$ cells from the broth were spread on LB agar plates containing Amp100 and kanamycin concentrations of the next selection level, and cultivated for colony counting. For example, cells from Kan100 broth would be spread on plates containing Kan200.

On each selection level, the culture broth was spread on LB agar plates containing Amp100 and incubated overnight. 30 single colonies were selected randomly and the dnaQ mutants carried were isolated and sequenced.

(ii) n-Butanol tolerance: evolution process for nbutanol tolerance of $E$. coli was similar to that described for kanamycin resistance. After a pre-cultivation in $0.5 \%$ of n-butanol, culture broth was stepwise transferred to LB media containing Amp100 and n-butanol with gradually increased concentrations from $0.625 \%$ to $1.25 \%$. Inoculation volume and cell densities for the three groups were adjusted to the same. On each n-butanol concentration, three transfers were performed before inoculation into a higher level. Each cultivation was performed for 48 hours. Tubes used in n-butanol tolerance evolution were sealed off with parafilm to avoid n-butanol evaporation.

(iii) Acetate tolerance: evolution process for acetate tolerance of $E$. coli was similar that described for nbutanol tolerance. Cultivations were performed in serial acetate concentrations of $0.06 \%, 0.075 \%, 0.09 \%$, and $0.105 \%$.

\section{Plasmid elimination}

The plasmid in the finally evolved strain was eliminated by serial transfer in LB medium without ampicillin but supplemented with the corresponding selective pressure (e.g., high concentrations of kanamycin or butanol or acetate). An appropriate amount of culture was spread on the agar plate with the same concentration of selective pressure before each transfer for colony visualization. Thirty single colonies were streaked on agar plate with ampicillin and those with ampicillin sensitivity were regard as the plasmid-cured ones. Typically 5-10 transfers are sufficient to isolate plasmid-cured strain maintaining the evolved phenotypes.

\section{Growth assay}

To evaluate tolerance of the GREACE generated cells, growth of the finally isolated mutant strains were analyzed under serial concentrations of n-butanol or acetate and compared with that of the wildtype E. coli strain. For growth assay, E. coli cells were cultivated overnight at $37^{\circ} \mathrm{C}$ in $\mathrm{LB}$ medium, and then diluted at a ratio of 1:100 into fresh LB media added with serial concentrations of n-butanol or acetate. Cultivations at $37^{\circ} \mathrm{C}, 200$ 
rpm were performed for 48 hours before calculation of $\mathrm{OD}_{600}$ to evaluate the cell densities.

\section{Shock experiment}

To explore tolerance and stability of $E$. coli BT12 strain under extreme lethal $\mathrm{n}$-butanol stress, shock experiments were performed with $2 \%(\mathrm{vol} / \mathrm{vol})$ of n-butanol. Procedure of the shock experiment was similar with previously introduced [58]. E. coli BT12 and E. coli DH5 $\alpha$ cells were cultivated overnight at $37^{\circ} \mathrm{C}$ in $\mathrm{LB}$ medium, and then diluted at a ratio of 1:100 with fresh LB medium and grown at $37^{\circ} \mathrm{C}$ to OD600 of $0.3 \sim 0.5$. The cultures were then diluted with $\mathrm{LB}$ to $\mathrm{OD}_{600}$ of 0.3 , and n-butanol was added to final concentrations of $2 \%$ (vol/ vol). After incubation at $37^{\circ} \mathrm{C}$ for 1 hour, the cultures were serially diluted, plated on LB agar plates and cultivated at $37^{\circ} \mathrm{C}$ overnight for photographing.

\section{Determination of mutation rate}

All of the selected $d n a Q$ mutators were retransformed into $E$. coli $\mathrm{DH} 5 \alpha$ strain with clean genetic background to determine the mutation rate. We calculated generation frequency of rifamycin resistant mutant cells to evaluate the mutation rates of the host cells, a method that has been applied widely in types of microbes $[59,60]$. E. coli cells transformed with specific dnaQ mutant were spread on LB agar plates and cultivated overnight in $37^{\circ} \mathrm{C}$. Three colonies were inoculated into fresh LB medium containing Amp100 and grown overnight. Approximately $10^{7}$ cells from the cultivation broth were spread on LB agar containing Amp100 with or without $100 \mu \mathrm{g} / \mathrm{ml}$ of rifamycin, and incubated in dark for 2 days. The rifamycin resistant colonies and total colonies were counted, and CFU data were used for determination of mutation rates.

\section{Additional file}

Additional file 1: Table S1. Primers used for plasmids and library construction in this work. Figure S1. Growth assay of E. coli strains carrying the pQ-dnaQ-KR5-2, pQ-dnaQ, and pUC18 plasmids in kanamycin concentrations of $15 \mu \mathrm{g} / \mathrm{ml}$ (A) and $100 \mu \mathrm{g} / \mathrm{ml}$ (B). Figure S2. Growth assay of the E. coli KR1 (pQ-dnaQ-KR5-2) and E. coli KR1 under kanamycin stress with (A) or without (B) Amp100. Figure S3. Shock experiment by $2 \%$ $\mathrm{n}$-butanol of the n-butanol tolerant strain E. coli BT12. Figure S4. Growth assay of the $\mathrm{n}$-butanol tolerant strain E. coli BT12 in serial n-butanol concentrations with or with pre-treatment.

\section{Abbreviations}

GREACE: Genome replication engineering assisted continuous evolution;

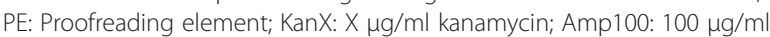
ampicillin; LB: Luria-Bertani.

\section{Competing interests}

The authors declare that they have no competing interests.
Authors' contributions

$G D L$ and $Z C$ designed and performed the research. GDL, ZC, and $Y L$ analyzed the data. GDL, ZC, YL, and YHM wrote the manuscript. $Y L$ and $Y H M$ supervised the project. All authors read and approved the final manuscript.

\section{Acknowledgements}

This work was supported by National Basic Research Program of China (973 program, 2011CBA00800), and National High Technology Research Program of China (863 program, 2012AA022100).

\section{Author details}

'CAS Key Laboratory of Microbial Physiological and Metabolic Engineering, Institute of Microbiology, Chinese Academy of Sciences, No. 1 West Beichen Road, Chaoyang District, Beijing 100101, China. ${ }^{2}$ University of Chinese Academy of Sciences, Beijing 100049, China. ${ }^{3}$ State Key Laboratory of Microbial Resources, Institute of Microbiology, Chinese Academy of Sciences, Beijing 100101, China.

Received: 4 June 2013 Accepted: 24 September 2013

Published: 27 September 2013

\section{References}

1. Patnaik R, Louie S, Gavrilovic V, Perry K, Stemmer WPC, Ryan CM, del Cardayre S: Genome shuffling of Lactobacillus for improved acid tolerance. Nat Biotechnol 2002, 20:707-712.

2. Alper H, Moxley J, Nevoigt E, Fink GR, Stephanopoulos G: Engineering yeast transcription machinery for improved ethanol tolerance and production. Science 2006, 314:1565-1568.

3. Stephanopoulos G, Alper H, Moxley J: Exploiting biological complexity for strain improvement through systems biology. Nat Biotechnol 2004, 22:1261-1267

4. Brynildsen MP, Liao JC: An integrated network approach identifies the isobutanol response network of Escherichia coli. Mol Syst Biol 2009, 5:277.

5. Nielsen J: Metabolic engineering. Appl Microbiol Biotechnol 2001, 55:263-283.

6. Petri R, Schmidt-Dannert C: Dealing with complexity: evolutionary engineering and genome shuffling. Curr Opin Biotechnol 2004, 15:298-304.

7. Meynial-Salles I, Soucaille P: Creation of new metabolic pathways or improvement of existing metabolic enzymes by in vivo evolution in Escherichia coli. Methods Mol Biol 2012, 834:75-86.

8. Auriol C, Bestel-Corre G, Claude JB, Soucaille P, Meynial-Salles I: Stress-induced evolution of Escherichia coli points to original concepts in respiratory cofactor selectivity. Proc Natl Acad Sci USA 2011, 108:1278-1283.

9. Meynial Salles I, Forchhammer N, Croux C, Girbal L, Soucaille P: Evolution of a Saccharomyces cerevisiae metabolic pathway in Escherichia coli. Metab Eng 2007, 9:152-159.

10. Parekh S, Vinci VA, Strobel RJ: Improvement of microbial strains and fermentation processes. Appl Microbiol Biotechnol 2000, 54:287-301.

11. Alper $\mathrm{H}$, Stephanopoulos $\mathrm{G}$ : Global transcription machinery engineering: A new approach for improving cellular phenotype. Metab Eng 2007, 9:258-267.

12. Park KS, Lee DK, Lee H, Lee Y, Jang YS, Kim YH, Yang HY, Lee SI, Seol W, Kim JS: Phenotypic alteration of eukaryotic cells using randomized libraries of artificial transcription factors. Nat Biotechnol 2004, 22:459.

13. Lee JY, Yang KS, Jang SA, Sung BH, Kim SC: Engineering butanol-tolerance in Escherichia coli with artificial transcription factor libraries. Biotechnol Bioeng 2011, 108:742-749.

14. Ochi K: From microbial differentiation to ribosome engineering Biosci Biotechnol Biochem 2007, 71:1373-1386.

15. Echols H, Goodman MF: Fidelity mechanisms in DNA replication. Annu Rev Biochem 1991, 60:477-511.

16. Maki H, Horiuchi T, Sekiguchi M: Structure and expression of the dnaQ mutator and the RNase $\mathrm{H}$ genes of Escherichia coli: Overlap of the promoter regions. Proc Natl Acad Sci USA 1983, 80:7137-7141.

17. Scheuermann R, Tam S, Burgers PMJ, Lu C, Echols H: Identification of the epsilon-subunit of Escherichia coli DNA polymerase III holoenzyme as the dnaQ gene product: a fidelity subunit for DNA replication. Proc Natl Acad Sci USA 1983, 80:7085-7089.

18. Kunkel TA, Bebenek R: DNA replication fidelity. Annu Rev Biochem 2000, 69:497-529

19. Cox EC, Horner DL: Structure and Coding Properties of a Dominant Escherichia-Coli Mutator Gene, Mutd. P Natl Acad Sci-Biol 1983, 80:2295-2299. 
20. Fijalkowska IJ, Schaaper RM: Mutants in the Exo I motif of Escherichia coli dnaQ: Defective proofreading and inviability due to error catastrophe. Proc Natl Acad Sci USA 1996, 93:2856-2861.

21. LeClerc JE, Li B, Payne WL, Cebula TA: High mutation frequencies among Escherichia coli and Salmonella pathogens. Science 1996, 274:1208-1211.

22. Matic I, Radman M, Taddei F, Picard B, Doit C, Bingen E, Denamur E, Elion J: Highly variable mutation rates in commensal and pathogenic Escherichia coli. Science 1997, 277:1833-1834.

23. Schaaper RM: Mechanisms of mutagenesis in the Escherichia coli mutator mutD5: role of DNA mismatch repair. Proc Natl Acad Sci USA 1988, 85:8126-8130

24. Atsumi S, Hanai T, Liao JC: Non-fermentative pathways for synthesis of branched-chain higher alcohols as biofuels. Nature 2008, 451:86-U13.

25. Dellomonaco C, Clomburg JM, Miller EN, Gonzalez R: Engineered reversal of the beta-oxidation cycle for the synthesis of fuels and chemicals. Nature 2011, 476:355-U131.

26. Shen CR, Lan El, Dekishima Y, Baez A, Cho KM, Liao JC: Driving Forces Enable High-Titer Anaerobic 1-Butanol Synthesis in Escherichia coli. Appl Environ Microb 2011, 77:2905-2915.

27. Atsumi S, Wu TY, Machado IM, Huang WC, Chen PY, Pellegrini M, Liao JC: Evolution, genomic analysis, and reconstruction of isobutanol tolerance in Escherichia coli. Mol Syst Biol 2010, 6:449

28. Reyes LH, Almario MP, Winkler J, Orozco MM, Kao KC: Visualizing evolution in real time to determine the molecular mechanisms of $n$-butanol tolerance in Escherichia coli. Metab Eng 2012, 14:579-590.

29. Zingaro KA, Papoutsakis ET: GroESL overexpression imparts Escherichia coli tolerance to i-, n-, and 2-butanol, 1,2,4-butanetriol and ethanol with complex and unpredictable patterns. Metab Eng 2013, 15:196-205.

30. Zingaro KA, Papoutsakis ET: Toward a Semisynthetic Stress Response System To Engineer Microbial Solvent Tolerance. Mbio 2012, 3:5.

31. Rutherford BJ, Dahl RH, Price RE, Szmidt HL, Benke PI, Mukhopadhyay A, Keasling JD: Functional genomic study of exogenous n-butanol stress in Escherichia coli. Appl Environ Microbiol 2010, 76:1935-1945.

32. Mao SM, Luo YAM, Zhang TR, Li JS, Bao GAH, Zhu Y, Chen ZG, Zhang YP, Li $Y$, Ma YH: Proteome reference map and comparative proteomic analysis between a wild type Clostridium acetobutylicum DSM 1731 and its mutant with enhanced butanol tolerance and butanol yield. J Proteome Res 2010, 9:3046-3061.

33. Chundawat SPS, Beckham GT, Himmel ME, Dale BE: Deconstruction of lignocellulosic biomass to fuels and chemicals. Annu Rev Chem Biomol Eng 2011, 2:121-145.

34. Mira NP, Palma M, Guerreiro JF, Sa-Correia I: Genome-wide identification of Saccharomyces cerevisiae genes required for tolerance to acetic acid. Microb Cell Fact 2010, 9:79.

35. Pena PV, Glasker S, Srienc F: Genome-wide overexpression screen for sodium acetate resistance in Saccharomyces cerevisiae. J Biotechnol 2013 164:26-33.

36. Hamdan S, Carr PD, Brown SE, Ollis DL, Dixon NE: Structural basis for proofreading during replication of the Escherichia coli chromosome. Structure 2002, 10:535-546.

37. Perrino FW, Harvey S, McNeill SM: Two functional domains of the epsilon subunit of DNA polymerase III. Biochemistry-Us 1999, 38:16001-16009.

38. Morrison A, Bell JB, Kunkel TA, Sugino A: Eukaryotic DNA polymerase amino acid sequence required for 3'-> 5' exonuclease activity. Proc Natl Acad Sci USA 1991, 88:9473-9477.

39. Blanco L, Bernad A, Salas M: Evidence favoring the hypothesis of a conserved 3'-5' exonuclease active-site in DNA-dependent DNApolymerases. Gene 1992, 112:139-144.

40. Giraud A, Matic I, Tenaillon O, Clara A, Radman M, Fons M, Taddei F: Costs and benefits of high mutation rates: adaptive evolution of bacteria in the mouse gut. Science 2001, 291:2606-2608.

41. Trobner W, Piechocki R: Competition between isogenic mutS and mut populations of Escherichia coli K12 in continuously growing cultures. Mol Gen Genet 1984, 198:175-176.

42. Gibson TC, Scheppe ML, Cox EC: Fitness of an Escherichia coli mutator gene. Science 1970, 169:686-688.

43. Taddei F, Radman M, MaynardSmith J, Toupance B, Gouyon PH, Godelle B: Role of mutator alleles in adaptive evolution. Nature 1997, 387:700-702.

44. Tenaillon O, Toupance B, Le Nagard H, Taddei F, Godelle B: Mutators, population size, adaptive landscape and the adaptation of asexual populations of bacteria. Genetics 1999, 152:485-493.
45. Horiuchi T, Maki H, Sekiguchi M: New conditional lethal mutator (dnaQ49) in Escherichia coli K12. Mol Gen Genet 1978, 163:277-283.

46. Tanabe K, Kondo T, Onodera Y, Furusawa M: A conspicuous adaptability to antibiotics in the Escherichia coli mutator strain, dnaQ49. Fems Microbiol Lett 1999, 176:191-196

47. Selifonova O, Valle F, Schellenberger V: Rapid evolution of novel traits in microorganisms. Appl Environ Microbiol 2001, 67:3645-3649.

48. Shimoda C, Itadani A, Sugino A, Furusawa M: Isolation of thermotolerant mutants by using proofreading-deficient DNA polymerase delta as an effective mutator in Saccharomyces cerevisiae. Genes Genet Syst 2006, 81:391-397.

49. Abe H, Fujita Y, Takaoka Y, Kurita E, Yano S, Tanaka N, Nakayama K: Ethanoltolerant Saccharomyces cerevisiae strains isolated under selective conditions by over-expression of a proofreading-deficient DNA polymerase delta. J Biosci Bioeng 2009, 108:199-204.

50. Loh E, Salk JJ, Loeb LA: Optimization of DNA polymerase mutation rates during bacterial evolution. Proc Natl Acad Sci USA 2010, 107:1154-1159.

51. Gonzalez-Ramos D, van den Broek M, van Maris AJ, Pronk JT, Daran JM: Genome-scale analyses of butanol tolerance in Saccharomyces cerevisiae reveal an essential role of protein degradation. Biotechnol Biofuels 2013, 6:48.

52. Sanchez RG, Karhumaa K, Fonseca C, Nogue VS, Almeida JRM, Larsson CU, Bengtsson O, Bettiga M, Hahn-Hagerdal B, Gorwa-Grauslund MF: Improved xylose and arabinose utilization by an industrial recombinant Saccharomyces cerevisiae strain using evolutionary engineering Biotechnol Biofuels 2010, 3:13.

53. Koppram R, Albers E, Olsson L: Evolutionary engineering strategies to enhance tolerance of xylose utilizing recombinant yeast to inhibitors derived from spruce biomass. Biotechnol Biofuels 2012, 5:32.

54. Jarboe LR, Grabar TB, Yomano LP, Shanmugan KT, Ingram LO: Development of ethanologenic bacteria. Adv Biochem Eng Biotechnol 2007, 108:237-261.

55. Jantama K, Zhang X, Moore JC, Shanmugam KT, Svoronos SA, Ingram LO: Eliminating Side Products and Increasing Succinate Yields in Engineered Strains of Escherichia coli C. Biotechnol Bioeng 2008, 101:881-893.

56. Zhang X, Jantama K, Moore JC, Shanmugam KT, Ingram LO: Production of L-alanine by metabolically engineered Escherichia coli. App/ Microbiol Biotechnol 2007, 77:355-366.

57. Stephanopoulos G: Metabolic engineering by genome shuffling - Two reports on whole-genome shuffling demonstrate the application of combinatorial methods for phenotypic improvement in bacteria. Nat Biotechnol 2002, 20:666-668.

58. Chen T, Wang J, Yang R, Li J, Lin M, Lin Z: Laboratory-evolved mutants of an exogenous global regulator, IrrE from Deinococcus radiodurans, enhance stress tolerances of Escherichia coli. Plos One 2011, 6:e16228.

59. Miller JH, Funchain $P$, Clendenin W, Huang T, Nguyen A, Wolff E, Yeung A, Chiang JH, Garibyan L, Slupska MM, Yang HJ: Escherichia coli strains (ndk) lacking nudeoside diphosphate kinase are powerful mutators for base substitutions and frameshifts in mismatch-repair-deficient strains. Genetics 2002, 162:5-13.

60. Sasaki M, Yonemura Y, Kurusu Y: Genetic analysis of Bacillus subtilis mutator genes. J Gen Appl Microbiol 2000, 46:183-187.

\section{doi:10.1186/1754-6834-6-137}

Cite this article as: Luan et al:: Genome replication engineering assisted continuous evolution (GREACE) to improve microbial tolerance for biofuels production. Biotechnology for Biofuels 2013 6:137. 